\title{
Transatlantica
}

Revue d'études américaines. American Studies Journal

\section{Regarder ceux qui regardent : Eudora Welty photographe}

Jean-Marc Victor

\section{OpenEdition}

1 Journals

\section{Édition électronique}

URL : https://journals.openedition.org/transatlantica/4559

DOI : $10.4000 /$ transatlantica.4559

ISSN : $1765-2766$

Éditeur

Association française d'Etudes Américaines (AFEA)

Référence électronique

Jean-Marc Victor, «Regarder ceux qui regardent : Eudora Welty photographe », Transatlantica [En ligne], 2 | 2009, mis en ligne le 01 février 2010, consulté le 01 février 2023. URL : http://

journals.openedition.org/transatlantica/4559; DOI : https://doi.org/10.4000/transatlantica.4559

Ce document a été généré automatiquement le 1 février 2023.

\section{(c) (i) (9)}

Creative Commons - Attribution - Pas d'Utilisation Commerciale - Pas de Modification 4.0 International - CC BY-NC-ND 4.0

https://creativecommons.org/licenses/by-nc-nd/4.0/ 


\title{
Regarder ceux qui regardent : Eudora Welty photographe
}

\author{
Jean-Marc Victor
}

Biographes et critiques ont largement souligné la concomitance, chez Eudora Welty, de la venue à l'écriture et de la pratique photographique au milieu des années $30^{1}$ : en 1936, elle obtient en même temps, et pour la première fois, la publication d'une de ses nouvelles ("Death of Traveling Salesman ») ainsi que la tenue d'une exposition, dans une galerie new-yorkaise, de ses photographies du Mississippi prises pour le compte de l'agence locale de la Works Progress Administration, qui l'emploie comme " publicity agent » de 1933 à 1936. Comme le souligne Suzanne Marrs avec raison : «What Eudora the photographer recorded on film, Eudora the writer recorded in memory » (Marrs 53-54).

2 Parmi la masse de clichés qu'elle produit à cette époque, et dont plusieurs inédits viennent d'être rendus accessibles au grand public par le récent ouvrage de Pearl Amelia McHaney, Eudora Welty as Photographer (voir bibliographie), je souhaite me pencher ici sur ceux qui, sans constituer à proprement parler une catégorie formellement identifiable, ont en commun de montrer des observateurs du monde qui les entoure (le Mississippi d'après la Grande Dépression) plus que ce monde lui-même : spectateurs en tous genres, auditoire anonyme de discours politiques, assistance fascinée par des numéros de fête foraine, foule guettant le passage de défilés, ou simples badauds témoins de la banalité du quotidien, ils occupent le premier plan d'un univers visuel où le regard de la photographe transite, semble-t-il, par d'autres regards que le sien, rendant ainsi marginal son objet, mais centrales ses modalités de médiation. S'ébauche ainsi à l'image une esthétique du détour qu'affinera bientôt la fiction en la transposant dans le champ d'une expression littéraire marquée par des stratégies d'obstruction et d'évitement sur un mode résolument anti-spectaculaire. Car avant la figure du lecteur dans le texte ${ }^{2}$, Welty eut recours à son pendant photographique : l'observateur dans l'image.

Bien que le spectacle lui-même ne soit pas absent de l'œuvre photographique de Welty constituée au gré de ses pérégrinations dans le Mississippi des années $30^{3}$, il est 
frappant de constater avec quelle fréquence l'objectif se tourne vers la foule plutôt que vers ce que ladite foule est venue voir. Ainsi, dans Mardi Gras / New Orleans (Photographs 143) [ill. 1], se postant à dessein au milieu de la rue où le défilé vient de passer, ou s'apprête à le faire, pour ne saisir que les postures des spectateurs dans toute leur diversité, Welty capte les marges de l'événement, son attente ou ses effets; mais dans une prolifération de cadres, de plans et de lignes de fracture, qui rappelle d'ailleurs le goût des photographes de la Nouvelle Vision pour le chaos urbain, elle escamote en lui tournant le dos le phénomène qui a généré le désir de voir. Elle ne fera rien d'autre dans la première partie de son roman The Optimist's Daughter (1972), située à la Nouvelle Orléans et dans laquelle l'un des personnages se plaint de ne rien voir des célébrations de Mardi Gras (Optimist 26) ${ }^{4}$. Dans cette photographie d'une foule qu'un cadrage décentré isole et retranche du spectacle qui justifie pourtant sa présence à l'image, Welty s'approprie déjà une pratique artistique qu'elle identifiera plus tard dans son essai « Looking at Short Stories » (1949), aussi bien en peinture qu'en littérature, sous le vocable de "halving ». Cette pratique consiste à laisser soit la moitié du tableau dans l'ombre (comme chez Goya, commente Welty dans cet essai), soit la moitié des constituants du récit fictionnel dans l'indétermination (l'action sans l'analyse de ses effets, ou inversement, comme chez Hemingway, suggère-t-elle encore) : « And it seems to be the halving that increases the story. » (Eye 90). Cette coupure entre ce qui sera montré et ce qui ne le sera pas est inhérente à l'image photographique comme prélèvement opéré dans le champ du réel ${ }^{5}$. Ici, la découpe ne conserve d'un tout que sa part anti-spectaculaire, tronquage qui deviendra presque signature dans l'ensemble de la fiction ${ }^{6}$. Parmi les spectateurs, trois au moins regardent en direction de l'objectif, signalant avec insistance la présence de l'opératrice et tout l'artifice qui, dans les coulisses de l'image, préside à sa réalisation. Ce qu'il y a à voir, n'est-ce pas, en conséquence, le geste même de la découpe plus que tout autre spectacle?

4 De même, dans Here it comes! / Jackson (Photographs 129) [ill. 2], des spectateurs enthousiastes et impatients ont pris d'assaut le toit d'un wagon pour mieux voir arriver ce «it» que le contenu de l'image n'élucide pas plus que son titre. La construction pyramidale, équivalent visuel d'un climax narratif, suggère que la satisfaction de l'attente ne saurait se traduire que par une retombée, alors que c'est bien ici que cela se passe, dans ce « here » étroitement cadré et pourtant décentré où tout se joue. Par effet de proximité et de déplacement, un hors-champ invisible justifie et fait exister le visible, lui donne sens dans une tension qui ne montre rien d'autre que le désir de voir, ou de savoir, exactement comme dans «June Recital ", nouvelle exemplaire du recueil The Golden Apples (Collected Stories), où deux enfants observent par la fenêtre les agissements énigmatiques de leur voisine d'en face.

Welty déploie par ailleurs toute une palette de stratégies visuelles pour confirmer que l'essentiel de son observation porte sur l'observateur, que son regard interroge le regard, que sa vision utilise le relais d'autres visions. Par exemple, Political Speech / Pontotoc (Photographs 62) [ill. 3] plonge l'orateur d'un meeting politique dans l'ombre des colonnes néo-classiques de quelque bâtiment officiel, dans la moitié supérieure de l'image, pour mieux surexposer les vêtements clairs d'une grande partie de l'assemblée, désignée ainsi dans la moitié inférieure comme véritable sujet d'observation. Parmi eux, un jeune homme se retourne vers nous comme pour vérifier que nous regardons dans la bonne direction et pour surprendre notre regard porté sur le regard d'autrui. 
6 Il arrive que le portrait de groupe se concentre sur quelques observateurs choisis et individualisés, comme c'est le cas pour Sideshow, State Fair / Jackson (Photographs 139) [ill. 4], où Welty, fidèle à la logique du "halving ", ne retient du spectacle que son effet, décliné en trois expressions sur le visage d'enfants témoins d'une attraction foraine totalement éclipsée par un étroit cadrage. Elle littéralise ainsi le titre du cliché, reléguant ce "side-show » qu'ils sont venus voir dans un hors-champ marginal, à moins qu'il ne s'agisse de désigner par ce titre ce qui, à côté du spectacle attendu, fait aussi spectacle. Amusement, circonspection, curiosité: la diversité des réactions qui s'inscrivent sur les traits des spectateurs, seul objet du regard photographique ${ }^{7}$, met précocement en abyme une réflexion sur les modalités de réception de l'image, à l'instar de toute l'œuvre à venir qui ne cessera de se dire ouverte, de désigner cette place à prendre qu'elle laissera bientôt à son lecteur dans une construction commune de l'objet artistique que sera la fiction :

at the other end of the writing is the reader. There is sure to be somewhere the reader, who is a user himself of imagination and thought, who knows, perhaps, as much about the need of communication as the writer.

Reader and writer, we wish each other well. Don't we want and don't we understand the same thing? A story of beauty and passion, some fresh approximation of human truth? (Eye 106)

7 C'est donc moins ce qu'il y a à voir que la manière de voir qui préoccupe la photographe dans ce type d'image, et moins la révélation d'un sens que le processus de son élaboration dans l'esprit de son lecteur, «à l'autre bout de l'écriture », qui sera au cœur des réflexions de l'auteur de fiction.

Même lorsque le spectacle apparaît à l'image, la présence insistante des spectateurs insérés dans le même cadre que l'objet de leur regard annule toute recherche d'illusion en renforçant l'artifice de la mise en scène, lequel désigne ouvertement le spectacle comme tel. Dans Hypnotized, State Fair / Jackson (Photographs 137) [ill. 5], par exemple, l'image se subdivise en trois plans qui ne sont rien d'autre que des types de représentation à des degrés divers : à l'arrière-plan, des affiches peintes, produits d'un art populaire qui a tant fasciné Walker Evans à la même époque, et où un sensationnalisme de pacotille le dispute à la candeur du trait; au centre, la représentation théâtrale à proprement parler, dont les accessoires rudimentaires soutiennent mal la comparaison avec la magie, même naïve, et l'inventivité fantaisiste des affiches du fond de scène; au premier plan, une rangée de jeunes spectateurs (sans doute les mêmes que pour le Sideshow précédent) figurant la nécessité d'un regard extérieur pour que la représentation prenne forme et sens. Plusieurs regards, notamment celui de l'hypnotiseur lui-même (assis à l'extrémité droite) et celui de deux des trois femmes debout sur la scène, ainsi que le corps à l'horizontale du cobaye (ou du complice ?) hypnotisé, pointent vers la gauche, comme pour inscrire sur une ligne médiane une sorte de visée, un axe de déplacement du regard en direction de l'aboyeur. Celui-ci débite son boniment, redoublant implicitement la présence du langage à l'image, déjà envahie par la graphie racoleuse des slogans qui, à rebours, se déroulent le long d'une autre ligne, quasiment parallèle à la précédente, mais orientée cette fois de gauche à droite (dans le sens de la lecture), faisant écho à deux regards orientés de même et qu'on pourrait dire récalcitrants : ceux de la femme en blanc sur la scène et de l'enfant retourné au premier plan. Ainsi sont mis à distance, dans une composition chaotique, les pouvoirs de fascination qu'exerce le triple artifice de l'image, du mot et 
de la mise en scène sur les lecteurs / spectateurs présents dans cette image, comme sur ceux de la fiction à venir, laquelle emploiera les mêmes outils pour parvenir à ses fins : une manière d'hypnose suscitée par une manière de boniment.

9 Un autre partage de l'image, binaire cette fois, s'opère dans The Rides, State Fair (McHaney 32) [ill. 6], où spectacle et spectateurs occupent à part égale l'espace photographique. La Grande Roue, rejetée à l'arrière-plan dans un flou aérien légèrement surexposé qui contribue à la dématérialiser, apparaît presque comme le fruit de l'imagination des trois femmes enlacées et solidement ancrées dans la moitié inférieure de l'image, alors que le grandiloquent lettrage «Royal American» du manège épelle sans y parvenir complètement un rêve ici privé de substantif, et partiellement de substance (un début de mot tronqué apparaît sur la bordure droite de l'image), ce qui le voue à un indéchiffrable flottement: nous regardons ces anonymes qui regardent, mais vouloir savoir à quoi elles rêvent reviendrait à anéantir ce rêve et la magie de l'instant ${ }^{8}$. Encore cette coupure, donc, cette scission entre la chose et son effet, le réel et son envers, la présence physique et le secret qu'elle recèle ${ }^{9}$. Encore cette ligne de texte, aussi, comme dans le cas précédent, qui traverse l'image telle une ligne d'horizon floue, mais qui accompagne cette fois le glissement du regard vers une sortie du cadre, dans une quête irrésolue du substantif manquant.

10 A l'inverse, dans Sideshow, setting up (McHaney 34) [ill. 7], c'est le premier plan, celui où passent les spectateurs, qui est flou, au moins en partie, tandis que le stand de foire que des ouvriers installent est aussi net que son ombre. Ici, l'illusion spectaculaire se montre pour ce qu'elle est, exhibant les grosses ficelles et autres filins qui la font tenir debout ainsi que les artisans qui la fabriquent de toutes pièces et qui occupent l'image en même temps que ceux qui les observent. Tout est aplani, le décor révèle les coulisses du rêve et le spectateur ne peut pas faire semblant de ne pas avoir vu qu'il y a montage, assemblage, trucage. Pour autant, l'ampleur de l'entreprise, l'ambition de la tromperie semblent justifier cet hommage photographique à la gloire d'une illusion dont toute la valeur est d'être captée en même temps par le regard des passants et par le nôtre. Dans l'inhabituelle sophistication de sa géométrie, le cliché évoque les expérimentations précisionnistes d'un Charles Scheeler ${ }^{10}$, mais dans une version où l'élément humain occuperait tout à coup le devant de la scène. La fascination de Welty photographe pour le signe écrit, autre trait qui la rapproche de Walker Evans, y transparaît déjà : dans le $\mathrm{E}$ du coin inférieur gauche, peint sur le stand, mais aussi dans le grand 0 inscrit dans l'ouverture centrale du décor, comme l'œil d'un dispositif optique où s'inscrit une figure humaine ${ }^{11}$, et flanqué d'une sorte de I en tôle ondulée qui le soutient de part et d'autre, tandis que dans une trouée du décor rappelant le caractère factice de cette façade sans profondeur s'inscrivent aussi, à peine discernables à côté du spectateur coiffé d'un chapeau, les principales lettres formant le titre "Royal American» de l'attraction voisine. Tout spectaculaire qu'il soit, le réel ne se donne donc pas comme un fragment déjà constitué mais comme un alphabet visuel, un texte mettant en scène son propre besoin de lecteurs capables de faire exister sa puissance d'illusion : « at the other end of the picture is the viewer ", est-on tenté de dire en paraphrasant Welty.

Plusieurs images postulent plus radicalement encore l'inexistence du réel en tant que donnée brute offerte à la prétendue transparence de la saisie photographique, préférant souligner dans leur construction même la nécessité d'une médiation subjective. Ce sont celles qui, sans aucun lien ni implicite ni explicite avec la moindre manifestation spectaculaire, se concentrent exclusivement sur l'acte de regarder dans 
sa banalité la plus dépouillée ou la plus quotidienne, pour soumettre dans le même temps cet acte à notre regard. At Livingston Park / Jackson (Photographs 56) [ill. 8] en est un exemple emblématique. Un enfant en costume de bain regarde à travers un grillage un plan d'eau où flottent les silhouettes floues et blanches d'une troupe de cygnes. L'objectif étant placé dans son dos et suffisamment bas pour adopter son point de vue et interposer le même quadrillage entre l'œil et le fond de la scène, nous le voyons qui voit et nous voyons ce qu'il voit. L'image n'est pas un échantillon prélevé dans la matière du monde mais plutôt comme une invitation à regarder un regard se poser sur cette matière. Cet enfant encore ruisselant vient juste de sortir de l'eau, comme le signalent la petite flaque à ses pieds et ses cheveux plaqués sur son crâne (autant de signes de l'existence d'un avant de l'image et d'une certaine épaisseur temporelle), ce qui implique aussi qu'il a appartenu à l'autre côté du grillage, comme nous tous, en tant qu'observateurs du réel, nous appartenons aussi à ce réel. Mais il s'en est extrait pour se poster à distance et l'image nous propose d'observer ce geste de recul et de contemplation. Ce que nous regardons avec lui, derrière lui, après lui (autre dimension de l'épaisseur temporelle inscrite dans le cadre), c'est une découpe opérée dans le réel et identifiable en plusieurs endroits de l'image : entre le haut et le bas de la rambarde, à l'intérieur des mailles triangulaires du grillage (répétées dans les deux triangles formés par les bras de part et d'autre de la tête de l'enfant, lesquels dessinent d'ailleurs un losange où s'inscrit cette tête, nouvel avatar d'une mise en abyme du dispositif de prise de vue), enfin dans l'étroit cadrage retenu par Welty. Ainsi, il n'y a pas le parc, il n'y a pas les cygnes sur le plan d'eau du parc, il y a la captation d'un regard offert à d'autres regards. Dans cette perspective, le grillage, écran à la fois réfractaire et projectif, si présent dans le plan médian de l'image, nous barre la vue en même temps qu'il postule l'existence de quelque chose à voir entre ses mailles.

12 Cet enfant annonce, bien sûr, la jeune narratrice de "A Memory " qui, au début de la nouvelle, observe à travers le cadre formé par ses doigts le lac, au milieu du parc, où elle vient de se baigner : «From my position I was looking at a rectangle brightly lit, actually glaring at me [...]. Ever since I had begun taking painting lessons, I had made small frames with my fingers, to look out at everything 》(Collected Stories 75). Si, de la photographie à la nouvelle, le rectangle a remplacé le triangle du maillage, le principe du dispositif de prise de vue reste identique: il se fonde sur cette obsession de la découpe qui donne à croire que le monde ne peut s'appréhender que déjà mis en cadre et déjà vu par d'autres. Cela ne l'empêche pas de faire déjà violemment retour dans l'univers du témoin ("glaring at me»), ce que confirmera le reste de la nouvelle (j'y reviendrai). La dette de cet incipit envers les arts visuels s'exprime symboliquement dans la double allusion à la peinture et à l'illustration (les arbres bordant le lac sont comparés à "the engraved thunderclouds surrounding illustrations in the Bible » [Collected Stories 75])..$^{12}$

13 Four boys leaning on a fence (McHaney 33) [ill. 9], qui montre quatre enfants accoudés à une barrière identique à celle de Livingston Park, regardant l'intérieur vide d'une grande cage (de base-ball ?) grillagée, se fonde sur une construction similaire tout en multipliant à l'intérieur de l'image les observateurs qui servent de relais au regard de l'observateur extérieur, et en redoublant la découpe du réel par l'entremise de deux maillages successifs, d'abord en triangle (la barrière) puis en losange (la cage). Ce qui attire ici le regard des enfants est invisible à l'image, car escamoté ou simplement inexistant : la capture de ce moment faible, de cet anti-« instant décisif », ne donne de nouveau à voir que les nombreuses médiations, à la fois humaines (observateurs mis en 
abyme) et techniques (prolifération des cadres), qui s'interposent entre le monde et ce qu'en retient l'image. Ce qui importe, c'est que ces quatre enfants sont à l'extérieur de la cage, de la même manière que le gamin de Livingston Park n'est plus dans l'eau, de même que les trois femmes dans The Rides ne sont plus, ou pas encore, dans les nacelles de la Grande Roue (à moins qu'elles n'y montent jamais) : dans tous les cas, l'image montre bien le fort pouvoir d'attraction, voire de fascination, du réel, qu'il soit spectaculaire ou banal, mais aussi la frontière qui nous sépare du réel. La trace photographique, dans laquelle il n'est plus possible de pénétrer physiquement autrement que par la seule entremise du regard, reproduit justement cette frontière, que matérialise encore le chemin qui nous sépare (en tant qu'observateurs de l'image) des observateurs dans l'image, même si nous sommes placés comme eux au bord d'une rangée de cailloux qui donne distance et profondeur à ce double effet de retrait. C'est trompeusement, donc, que la photographie nous offre le monde : à dire vrai, elle nous en prive en lui substituant une image et expose cette privation, ce retranchement, que Welty met en scène sur un mode réflexif en choisissant pour sujet tous ces observateurs.

Il suffit de se pencher sur Courthouse steps / Fayette (Photographs 64) [ill. 10] pour se convaincre que l'enjeu esthétique de telles images repose souvent sur la simple coprésence du regard de la photographe et de regards autres devant un non-événement: ici, une scène de rue où rien ne passe (les automobiles qu'aurait pu figer la saisie photographique sont déjà à l'arrêt) et rien ne se passe. Le vide narratif renvoie l'observateur à une réflexion sur des modalités de représentation plutôt que sur un contenu (documentaire, anecdotique, etc.): soulignement des grandes polarités constitutives du cliché photographique (ombre et lumière, mouvement ascendant ou descendant des diagonales créant un effet de profondeur, alignement des véhicules orientés vers la gauche ou la droite comme pour suggérer des modalités de sortie du cadre et un affranchissement de l'arbitraire du cadrage), fascination pour les rythmes générés par un système de réduplication (des marches, des dalles du trottoir, des chapeaux, des autos) qui évoque la reproductibilité au cœur du photographique. D'ailleurs, l'enchâssement des regards (celui des trois témoins dans celui de la photographe, qui lui-même s'offre au nôtre) s'opère lui aussi sur un mode reproductif, mais au gré de décentrements successifs qui en font toute la force: nous sommes derrière les trois hommes, mais légèrement de côté, de sorte que notre regard forme avec le leur un angle irréductible qui souligne un glissement de la perspective, unique déplacement (et unique événement) dans le moment faible de cette scène statique. Car si Welty écrivain pratique la relativité et l'éclatement du point de vue, ce n'est pas seulement parce qu'elle a retenu la leçon des grands modernistes qui l'ont précédée dans le champ de la littérature (Woolf, Faulkner, entre autres); c'est aussi derrière le viseur de son appareil photographique qu'elle y a pris goût.

«Before there is meaning, there has to occur some personal act of vision " (Eye 136), affirme Welty dans son essai «Words into Fiction » (1965): il ne fait pas de doute que cet impératif qui s'impose à la fiction préside aussi à l'exercice précoce de sa pratique photographique, tout particulièrement dans les clichés abordés dans les limites de ce court essai. Pour personnelle qu'elle soit, cette vision se construit en transitant par d'innombrables relais, dont la figure de l'observateur inscrit dans l'image n'est qu'un avatar. Man and woman meeting (McHaney 55) [ill. 11] nous donne à voir, en guise de conclusion, une sorte d'emballement de ce principe d'élaboration de la vision et du sens qui en découle. Dans un flou qui sied à la fugitive violation d'une intimité indûment 
exposée, dans une prolifération de cadres dans le cadre (panneaux, fenêtres), sur fond de lettrage tronqué mais dessinant un axe qui s'enfonce dans la profondeur de la photographie, appelant l'investissement extérieur d'un lecteur / observateur « à l'autre bout de l'image / texte " pour donner sens à ce qui n'est que fragment, des regards se croisent dans un complexe réseau de transactions secrètes où des mains se frôlent comme s'échangent les œillades ${ }^{13}$. C'est un couple : elle baisse le regard vers le geste qu'il esquisse de la main ; lui, comme s'il se sentait surpris, regarde à l'arrière-plan des femmes témoins de leur rencontre. C'est un triangle : ces femmes regardent vers eux. C'est un carré: la photographe regarde le couple, l'homme regarde les femmes à l'arrière-plan, qui regardent le couple mais sont aussi tournées vers la photographe, qui les regarde elles aussi. Et c'est nous qui les regardons regarder, nous encore qui, indirectement, au-delà de la photographe prise la main sur le déclencheur, sommes vus en train de regarder, pris « l'œil sur l'image ».

Car l'image fait toujours retour sur qui la regarde, et c'est aussi le cas dans la fiction : la narratrice de " A Memory ", après avoir transmué le monde en image à force de le faire entrer dans le cadre formé par ses doigts, se sent à son tour observée par l'un des baigneurs qui appartenaient à l'image par elle construite, retournant ainsi le regard de l'observatrice sur sa propre personne: «He even looked at me, and included me. Looking back, stunned, I wished that they were all dead ( (Collected Stories 78). De même sommes-nous inclus, happés, absorbés, dans le dialogue des regards qui anime toutes ces photographies d'observateurs, appelés à prendre la place qui nous y est laissée, à la fois ouverte et intime, parmi ces êtres dont la mortalité est aussi la nôtre, mais dont le regard n'a pas péri et continue d'interroger notre manière de voir l'image.

\section{BIBLIOGRAPHIE}

CHOUARD, Géraldine \& PITAVY, Danièle. Eudora Welty and the Poetics of the Body. Etudes faulknériennes, Volume 5, Presses Universitaires de Rennes, 2005.

DURAND, Régis. Le Regard pensif. Paris : La Différence, 2002.

MARRS, Suzanne. Eudora Welty. A Biography. Orlando : Harcourt, 2005.

McHANEY, Pearl Amelia (ed.). Eudora Welty as Photographer. Jackson : University Press of Mississippi, 2009.

WELTY, Eudora. The Optimist's Daughter. London : Virago, Modern Classics, 1984 (1972).

--. The Eye of the Story: Selected Essays and Reviews. London : Virago, 1987 (1979).

--. The Collected Stories of Eudora Welty. New York: Harcourt Brace Jovanovich, 1980.

--. One Writer's Beginnings. New York : Warner, 1991 (1984).

--. Photographs. Jackson : University Press of Mississippi, 1989.

--. Early Escapades. Edited by Patti Carr Black. Jackson : University Press of Mississippi, 2005. 


\section{NOTES}

1. Cf "Picturing the Body", Eudora Welty and the "Poetics of the Body. Géraldine Chouard \& Danièle Pitavy, eds, Etudes faulknériennes, Volume 5, Presses Universitaires de Rennes, 2005, 19-55.

2. L'acte de lire est diversement mis en scène dans l'œuvre littéraire de Welty, depuis «A Piece of News » jusqu'à One Writer's Beginnings, en passant par "The Demonstrators » et The Optimist's Daughter.

3. Voir par exemple les photographies de chars pavoisés lors de la «State Fair Parade » de Jackson (Photographs 77-81).

4. Plus largement, plusieurs moments essentiels de la séquence diégétique du roman seront également tronqués ou éclipsés, conformément à une stratégie de diversion surtout optique, parfois sonore : le face-à-face entre Fay et le juge McKelva avant sa mort à l'hôpital (Optimist 32), cette mort elle-même (40) ainsi que l'enterrement qui s'ensuit, dont sa fille Laurel ne perçoit que des bribes lors de l'oraison funèbre, couverte par les bruits parasites de la nouvelle route voisine (92).

5. C'est ce que rappelle Régis Durand lorsqu'il écrit que toute photographie « exhibe le bord vif d'une coupure » (Durand 26).

6. Où sont passées, par exemple, «the silver apples of the moon » dans The Golden Apples (1949), recueil dont le titre cite (en les tronquant) les derniers vers du poème de Yeats « The Song of the Wandering Aengus » : "The silver apples of the moon, / The golden apples of the sun »?

7. Dans un registre bien plus cruel, Weegee poussera jusqu'au cynisme cette scrutation du regard des témoins dans toute leur variété dans Their First Murder (1941), où il photographie un groupe d'enfants qui se pressent sur le lieu d'un crime pour voir le mort laissé, là encore, hors champ par le biais d'un cadrage serré. Un peu comme Welty avant lui, mais sur le mode grinçant qui lui est propre, il choisit de braquer l'objectif sur ces jeunes spectateurs qui font spectacle à la place du spectacle et qui, d'ailleurs, se l'approprient jusque dans le brutal possessif du titre.

8. Dans son essai intitulé «The Observing Eye », Pearl Amelia McHaney cite Welty évoquant la part de rêve inscrite dans cette photographie : «the Ferris Wheels should be brought out to be right in front of the girls' eyes to dazzle them like an impossible dream » (McHaney 18).

9. On songe à la description de la tête de Jamie, le commis jardinier, dans « A Curtain of Green » : «the bowed head holding so obviously and so fatally its ridiculous dream » (Collected Stories 110). La manière dont la question de l'appartenance raciale est susceptible, dans les deux cas, de compliquer les enjeux, pourrait faire l'objet d'une autre étude. Qu'il s'agisse de vision réelle ou onirique, j'ajouterai seulement qu'il y a, dans la nouvelle comme dans cette photographie (et beaucoup d'autres) une intéressante mise en abyme du regard noir dans le regard blanc, conduisant toujours à une relative opacité : comme Mrs. Larkin en embuscade derrière Jamie, la photographe blanche qui se poste dans le dos de ces trois femmes noires sait déceler la présence d'un rêve qui les habite, mais ne saurait en donner la clé.

10. On se souvient notamment de la série de photographies de l'usine Ford de Detroit prises par Scheeler en 1927, en particulier Criss-Crossed Conveyors, Ford Plant, où l'on retrouve, dans un contexte non plus forain mais industriel, un dialogue similaire entre courbes (des cuves) et lignes (des structures métalliques de l'usine), entre obliques (des tapis roulants) et verticales (des cheminés dressées comme des grandes orgues).

11. On peut y voir une miniature réflexive tendue à l'objectif, comme un miroir qui retournerait le regard photographique sur lui-même.

12. Patti Carr Black montre dans Early Escapades (voir bibliographie) comment Welty s'essaya dans sa jeunesse à l'art de l'illustration.

13. Ces regards tournoyants, réversibles, échangés dans un lieu public, évoquent le virtuose glissement des points de vue dans l'incipit de "No Place for You, My Love ", autre brève rencontre entre un homme et une femme à la Nouvelle Orléans : il la voit ainsi, elle le voit comme 
ça, elle se voit tout autre, d'autres encore autour d'eux les voient étrangers à l'endroit (Collected Stories 465-67). Le texte de la nouvelle mettra à l'épreuve ces diverses représentations.

INDEX

Thèmes : Trans'Arts

AUTEUR

JEAN-MARC VICTOR

Université Paris - Sorbonne 